\title{
An NTU Cooperative Game Theoretic View of Manipulating Elections
}

\author{
Michael Zuckerman ${ }^{1}$, Piotr Faliszewski ${ }^{2}$, Vincent Conitzer ${ }^{3}$, and Jeffrey S. \\ Rosenschein $^{1}$ \\ 1 School of Computer Science and Engineering, The Hebrew Univ. of Jerusalem, Israel. \\ \{michez, jeff\}@cs.huji.ac.il \\ 2 AGH University of Science and Technology, Kraków, Poland. faliszew@agh.edu.pl \\ 3 Department of Computer Science, Duke University Durham, NC 27708, USA. \\ conitzer@cs.duke.edu
}

\begin{abstract}
Social choice theory and cooperative (coalitional) game theory have become important foundations for the design and analysis of multiagent systems. In this paper, we use cooperative game theory tools in order to explore the coalition formation process in the coalitional manipulation problem. Unlike earlier work on a cooperative-game-theoretic approach to the manipulation problem [2], we consider a model where utilities are not transferable. We investigate the issue of stability in coalitional manipulation voting games; we define two notions of the core in these domains, the $\alpha$-core and the $\beta$-core. For each type of core, we investigate how hard it is to determine whether a given candidate is in the core. We prove that for both types of core, this determination is at least as hard as the coalitional manipulation problem. On the other hand, we show that for some voting rules, the $\alpha$ - and the $\beta$-core problems are no harder than the coalitional manipulation problem. We also show that some prominent voting rules, when applied to the truthful preferences of voters, may produce an outcome not in the core, even when the core is not empty.
\end{abstract}

\section{Introduction}

Voting constitutes a natural methodology for a group of agents to make a joint decision in spite of (possibly) conflicting preferences. Unfortunately, voting and elections are not a universal, perfect solution to preference aggregation problems. For example, the classic result of Gibbard and Satterthwaite $[9,13]$ says that in sufficiently general settings, any reasonable voting rule may lead to a situation where some voter(s) are better off by casting votes different from their true preferences (this is called manipulation or strategic voting). One of the most influential ideas regarding the computational study of elections, due to Bartholdi, Orlin, Tovey, and Trick [4,3], was to study the computational complexity of computing manipulative votes. The rationale behind it was that if planning a manipulation were computationally hard, then in practice voters would not be able to vote strategically (see the surveys of Faliszewski, Hemaspaandra, and Hemaspaandra [7] and of Faliszewski and Procaccia [8] for a detailed overview of this approach and for two viewpoints regarding its applicability).

Formally, in the coalitional manipulation problem, introduced by Conitzer, Sandholm, and Lang [5], the voters are divided into two groups, the manipulators and the 
nonmanipulators. The votes of the nonmanipulators are assumed to be known, and the problem is to determine whether the manipulators can use their votes to achieve a given goal. The goal is either to ensure that some preferred candidate wins (the constructive variant) or to ensure that some despised candidate does not win (the destructive variant). Further, we obtain different flavors of the problem depending on whether the votes are weighted or not, which voting rule is used, etc. Some results on coalitional manipulation can be found in $[17,15,14,16]$; see also surveys $[8,7]$.

Let us focus on unweighted constructive coalitional manipulation. If all the manipulators have identical preferences, this is exactly the problem that they want to be able to solve - they would first try to ensure their most preferred candidate's victory; then, if that were impossible, they would try the second best one, third best one, and so on, until they would either find a successful manipulation or determine that they cannot do better than electing the truthful winner.

Nonetheless, generally manipulators do not have identical preferences. In this case, the manipulators may still work together, but it is much less clear which candidate they should try to promote (even ignoring computational considerations). While they may all agree that they would prefer a different winner than the truthful one, deciding which candidate to support is a whole new game that they need to play among themselves. (To push our scenario to the limit, consider a situation where all the voters are manipulators.)

In this paper, we take the viewpoint of cooperative game theory to solve such games among the manipulators, and study computational aspects of the relevant solution concepts. As in most of the literature on voting, we assume that the agents do not have the ability to make or receive payments, so that we are in the nontransferable utility (NTU) case of cooperative game theory. (Recently, Bachrach, Elkind, and Faliszewski [2] studied a similar problem in the transferable utility setting, and obtained results linking coalitional manipulation [5] and bribery [6] problems with their cooperative gametheoretic model.) Moreover, in this setting, what one (sub)coalition of manipulators can achieve depends on the actions (votes) of the manipulators outside the coalition. We consider two different ways of addressing this_-via the standard notions of the $\alpha$ and the $\beta$-core [12].

\section{Preliminaries}

Let us now define the basic notions of (computational) social choice theory and coalitional game theory, as used in this paper.

An election $E$ is a triple $(C, V, \mathcal{P})$, where $C=\left\{c_{1}, \ldots, c_{m}\right\}$ is the set of candidates, $V=\{1, \ldots, n\}$ is the set of voters, and $\mathcal{P}=\left(P_{1}, \ldots, P_{n}\right)$ is a preference profile of voters in $V$. That is, each voter $i, 1 \leq i \leq n$, is associated with preference order $P_{i}$ from $\mathcal{P}$. A preference order is a linear order over the candidates in $C$. We will sometimes write $\succ_{i}$ instead of $P_{i}$. We write $L(C)$ to denote the set of all linear orders over $C$. Let $U$ be some subset of $V$. By $\mathcal{P}_{U}$ we mean $\left(P_{i}\right)_{i \in U}$ and by $\mathcal{P}_{-U}$ we mean $\left(P_{i}\right)_{i \notin U}$. Using standard notational conventions, we have $\mathcal{P}=\left(\mathcal{P}_{U}, \mathcal{P}_{-U}\right)$.

A voting rule $\mathcal{R}$ is a function that, given election $E$ as input, returns one of the candidates, denoted $\mathcal{R}(E)$, as the election's winner. (We assume ties are resolved by some 
simple tie-breaking rule and that the manipulators, unwilling to rely on tie-breaking, always seek unique winners; we point the reader to the work of Obraztsova, Elkind, and Hazon [11], and Obraztsova and Elkind [10] for a detailed discussion of tie-breaking in voting manipulation.) We focus on the following (families of) voting rules:

Positional scoring rules. Let $s=\left(s_{1}, \ldots, s_{m}\right)$ be a vector of non-negative integers, such that $s_{1} \geq s_{2} \geq \ldots \geq s_{m}$. For each voter, a candidate receives $s_{1}$ points if it is ranked first by the voter, $s_{2}$ points if it is ranked second, etc. The score of a candidate is the total number of points the candidate receives. The winner is the candidate with the maximum score. The scoring rules which we will consider here are k-approval, where $s=(1, \ldots, 1,0, \ldots, 0)\left(s_{1}=\ldots=s_{k}=1 ; s_{k+1}=\ldots=\right.$ $\left.s_{m}=0\right)$, Plurality, where $s=(1,0, \ldots, 0)$, and Borda, where $s=(m-1, m-$ $2, \ldots, 0)$.

Plurality with Runoff. In this rule, a first round eliminates all candidates except the two with the highest plurality scores. The second round determines the winner between these two by their pairwise election.

Simplified Bucklin. A candidate $c$ 's Bucklin score is the smallest number $k$ such that more than half of the votes rank $c$ among the top $k$ candidates. The winner is the candidate that has the smallest Bucklin score. ${ }^{4}$

We use the term (coalitional) manipulation to refer to a situation where a voter (a group of voters) casts votes not according to his (their) true preferences, but rather to obtain some goal. It is one of the best-studied forms of strategic behavior in elections (see the surveys $[8,7])$. The definition below is taken from the paper of Bachrach, Elkind, and Faliszewski [2], which itself is inspired by the definition of Conitzer, Sandholm, and Lang [5].

Definition 1. For any voting rule $\mathcal{R}$, an instance $I=(E, S, c)$ of the $\mathcal{R}$-COALITIONAL MANIPULATION problem is given by an election $E=(C, V, \mathcal{P})$, a set of manipulators $S, S \cap V=\emptyset$, and a distinguished candidate $c \in C$. It is a "yes"-instance if there is a vector $\mathcal{P}_{S}=\left(P_{i}\right)_{i \in S} \in(L(C))^{|S|}$ such that $\mathcal{R}\left(\mathcal{P}, \mathcal{P}_{S}\right)=c$, and a "no"-instance otherwise.

Note that in the traditional definition of coalitional manipulation the manipulators, unlike honest voters, do not have preferences over the candidates; they simply want to get a particular candidate elected. Thus, this definition eliminates the problem of deciding which candidates the manipulators should support by making it external to the setting.

\section{Manipulation Model}

The goal of this paper is to study the unweighted constructive coalitional manipulation problem in the setting in which not all manipulators have identical preferences. Specifically, our focus is on the computational complexity of deciding which candidates the manipulators may support in a stable way, without breaking the coalition.

\footnotetext{
${ }^{4}$ The Nonsimplified Bucklin rule additionally breaks ties by the number of votes that rank a candidate among the top $k$ candidates. In computational social choice it is common to focus on Simplified Bucklin instead of its full variant.
} 
Formally, we consider the following setting. We are given an election $E=(C, V, \mathcal{P})$ where some of the voters are truthful and some are willing to manipulate. We denote the set of possible manipulators by $M$ and we will refer to them as colluders. In our model the remaining voters, those in $H=V \backslash M$, are honest voters who vote truthfully. We assume that the manipulators have a way of communicating with one another (this is a standard assumption, though typically it is not mentioned explicitly). We ask which candidate the colluders should support. Naturally, the answer to this question depends strongly on the attitudes that the colluders have towards one another, and on the way they expect one another to behave. We consider two settings, depending on how the colluders react to the breaking out of the coalition by some subset $M^{\prime}$ of $M$ : (a) the pessimistic model, where players in $M^{\prime}$ want to succeed irrespective of the reaction of players in $M \backslash M^{\prime}$, and (b) the adaptive model, where players in $M^{\prime}$ can pick their votes depending on the votes of players in $M \backslash M^{\prime}$.

Definition 2. Let $\mathcal{R}$ be a voting rule and let $E=(C, V, \mathcal{P})$ be an election with colluder set $M$. Fix a candidate $c \in C$ and a coalition $S \subseteq M$.

1. We say that $c$ is feasible for $S$ if there is a profile $\mathcal{P}_{S}^{\prime}$ such that $\mathcal{R}\left(\mathcal{P}_{S}^{\prime}, \mathcal{P}_{-S}\right)=c$.

2. We say that $c$ is $\alpha$-feasible for a coalition $S$ if there is a preference profile $\mathcal{P}_{S}^{\prime}$ such that for all preference profiles $\mathcal{P}_{M \backslash S}^{\prime \prime}$ it holds that $\mathcal{R}\left(\mathcal{P}_{S}^{\prime}, \mathcal{P}_{M \backslash S}^{\prime \prime}, \mathcal{P}_{-M}\right)=c$.

3. We say that $c \in C$ is $\beta$-feasible for a coalition $S \subseteq M$ if for all preference profiles $\mathcal{P}_{M \backslash S}^{\prime}$ there exists a preference profile $\mathcal{P}_{S}^{\prime \prime}$ such that $\mathcal{R}\left(\mathcal{P}_{S}^{\prime \prime}, \mathcal{P}_{M \backslash S}^{\prime}, \mathcal{P}_{-M}\right)=c$.

We denote the set of all candidates that are feasible (respectively, $\alpha$-feasible, $\beta$-feasible) for $S$ by $F(S)$ (respectively, $F_{\alpha}(S), F_{\beta}(S)$ ).

As a side remark, it is easy to see that $F(S)$ is never empty as it always contains $\mathcal{R}(E)$; but $F_{\alpha}(S)$ and $F_{\beta}(S)$ may be empty.

Using our feasibility notions, we can adapt the notions of $\alpha$ - and $\beta$-core to our setting [12].

Definition 3. Let $E=(C, V, \mathcal{P})$ be an election, $M$ be a subset of $V, \mathcal{R}$ be a voting rule, and $c$ be a candidate. We say that c belongs to the $\alpha$-core (respectively, $\beta$-core) if $c \in F(M)$ and there is no candidate $c^{\prime} \in C$ and non-empty coalition $W^{\prime} \subseteq M$ such that (a) $c^{\prime} \in F_{\alpha}\left(W^{\prime}\right)$ (respectively, $c^{\prime} \in F_{\beta}\left(W^{\prime}\right)$ ), and $(b)$ each voter in $W^{\prime}$ prefers $c^{\prime}$ to $c$.

One can see that these notions are related to the notion of strong Nash equilibrium (SNE) in the game played by the colluders. Recall that an SNE is a Nash equilibrium in which no coalition, taking the actions of its complements as given, can cooperatively deviate in a way that benefits all of its members [1]. In the context of SNE, we have the following scenario: the colluders have agreed upon some voting profile. The deviating coalition can privately communicate; when a coalition coordinates a deviation, the remaining players are unaware of it, so they stick to their agreed-upon strategies. In this model, it is relatively easy for a sub-coalition to break off since the deviating colluders know in advance how the rest of the colluders will vote. On the other hand, the $\alpha$-core and the $\beta$-core model an election in which it is non-trivial for a subgroup of manipulators to break off from the coalition. (Consider, e.g., an election on choosing 
an acceptable debt level for a country and the manipulators being MPs from the ruling party. Even if they personally disagreed with some particular proposed debt level, they would need to obtain very strong support before breaking off from the coalition.) Here, if a subcoalition breaks off then it has to be ready to face every possible reaction of the colluders they abandon.

The difference between the $\alpha$-core and the $\beta$-core is that in the case of the $\beta$-core the splitting subcoalition knows that it will know the remaining colluders' votes before having to cast their own. (In the parliamentary example from the previous paragraph, this means that MPs leaving the ruling party know they will be asked about their vote on the debt issue only after the ruling party will be.)

The above intuitions are supported by the observation that, by definition, if $\mathcal{P}_{M}^{\prime}$ is SNE then $c=\mathcal{R}\left(\mathcal{P}_{H}, \mathcal{P}_{M}^{\prime}\right) \in \beta$-core, and $\beta$-core $\subseteq \alpha$-core. (To see this, note that if for $c^{\prime} \in C \backslash\{c\}$, and $W^{\prime} \subseteq M$ such that everybody in $W^{\prime}$ prefers $c^{\prime}$ to $c, c^{\prime} \in F_{\beta}\left(W^{\prime}\right)$ then $W^{\prime}$ can deviate from $\mathcal{P}_{M}^{\prime}$ to make $c^{\prime}$ a winner, and so $\mathcal{P}_{M}^{\prime}$ is not a SNE; and if for some candidate $c^{\prime}$ and set $W^{\prime} \subseteq M$ it holds that $c^{\prime} \in F_{\alpha}\left(W^{\prime}\right)$ then $c^{\prime} \in F_{\beta}\left(W^{\prime}\right)$.) That is, settings where the $\alpha$-core is an appropriate model are more stable than those where colluders do not counteract deviations by other colluders.

It is interesting that there are prominent voting rules and preference profiles for which the non-empty $\alpha$-core $/ \beta$-core does not contain the truthful winner.

Example 4. Consider a Borda election with candidate set $C=\{a, b, c, d, e, f\}$, and the following preference profiles (the numbers in parentheses are the serial numbers of the voters): (1) $f \succ d \succ b \succ c \succ a \succ e$, (2) $a \succ f \succ b \succ c \succ d \succ e$, (3) $b \succ f \succ d \succ c \succ a \succ e$, and (4) $d \succ b \succ f \succ a \succ e \succ c$.

Here we have one honest voter (1), and the rest are the colluders. In the above election, $f$ wins. Now consider the set $W_{b f}=\{3,4\}$. If (3) votes $b \succ e \succ c \succ$ $a \succ d \succ f$, and (4) votes $b \succ a \succ e \succ c \succ d \succ f$, then no matter how voter $(2)$ votes, $b$ wins the election. Hence $b \in F_{\alpha}\left(W_{b f}\right) \Rightarrow f \notin \alpha$-core. We claim that $b \in \beta$-core (and hence $b \in \alpha$-core). Indeed, $b$ is the Condorcet winner among the colluders. Hence, if the two colluders who prefer $b$ to some candidate $x \in\{a, c, d, e, f\}$, vote $b \succ e \succ c \succ a \succ d \succ f$ and $b \succ a \succ e \succ c \succ d \succ f$, then no matter how the third colluder votes, $b$ wins the election. Hence, no other candidate is $\beta$-feasible for the coalition that prefers him, which implies $b$ is in the $\beta$-core.

We also have a similar example for Maximin, but we omit it due to space restrictions. ${ }^{5}$

To study our core notions computationally, we need to define an appropriate decision problem.

Definition 5. For a voting rule $\mathcal{R}$, an instance $I=(E, M, c)$ of the $\mathcal{R}$ - $\alpha$-CORE (respectively, $\mathcal{R}$ - $\beta$-Core) problem, is given by an election $E=(C, V, \mathcal{P})$, a set of colluders $M \subseteq V$ and a candidate $c \in C$. It is a "yes"-instance if $c$ is in the $\alpha$-core (respectively, $\beta$-core) of the corresponding game among the manipulators, and a "no"-instance otherwise.

\footnotetext{
${ }^{5}$ See ftp://ftp.cs.huji.ac.il/users/jeff/winellzuckermanfull.pdf for a version of this paper containing the example.
} 


\section{Main Results}

In this section we present our main computational results. Namely, we provide a computational analysis of the notions of the $\alpha$ - and $\beta$-cores. We first provide a connection between our notions of the core and the standard problem of coalitional manipulation.

Theorem 6. Given an election $E=(C, V, \mathcal{P})$, a set $M \subseteq V$, a voting rule $\mathcal{R}$ and a candidate $c \in C$, the problem of determining whether $c$ is in the $\alpha$-or $\beta$-core is at least as hard as the corresponding coalitional manipulation problem.

Proof. We show a reduction from the coalitional manipulation problem. Given a voting rule $\mathcal{R}$ and an instance $I=(E, S, c)$, where $E=(C, V, \mathcal{P})$, of the $\mathcal{R}$-Coalitional Manipulation problem, we construct the core problem in the following way. We set $E^{\prime}=\left(C, V^{\prime}, \mathcal{P}^{\prime}\right)$ where $V^{\prime}=V \cup S, \mathcal{P}^{\prime}=\mathcal{P} \cup \mathcal{P}_{S}^{\prime}$, and $\mathcal{P}_{S}^{\prime}$ is a profile of voters in $S$ where $c$ is ranked first in all the ballots, and the rest of the candidates are ranked in some arbitrary order. We set $M=S$. There exists a manipulation by voters in $S$ making $c$ win in the coalitional manipulation problem if and only if $c$ is in the core (either $\alpha$ or $\beta$ ) of the core problem (which is defined by the instance $\left(E^{\prime}, M, c\right)$ ), since here $c$ is in the core if and only if $c$ is feasible for the coalition $M$ (as there is no non-empty coalition $W^{\prime}$ where the voters in $W^{\prime}$ prefer $c^{\prime}$ to $c$ ).

However, could it be that the $\alpha$ - or the $\beta$-core problems are strictly harder than the coalitional manipulation problem? We will see that for many voting rules, the answer is "no". Due to Theorem 6, we focus on those voting rules for which the coalitional manipulation problem is polynomial-time solvable.

Theorem 7. Let $R$ be a positional scoring rule with scoring vector $s=\left(s_{1}, \ldots, s_{m}\right)$ such that the $R$-Coalitional Manipulation problem is polynomial-time solvable. Then there exists a polynomial-time algorithm solving the $R$ - $\alpha$-Core problem.

Proof. Suppose we are given an instance $I=(E, M, c)$ of the $R$ - $\alpha$-Core problem, where $E=(C, V, \mathcal{P})$. Suppose w.l.o.g. that $s_{m}=0$. The algorithm first checks whether $c \in F(M)$ by solving the $R$-Coalitional Manipulation problem. Then it goes over all the other candidates. For each candidate $x \neq c$ it checks what is the maximal set $W_{x c} \subseteq M$ of the colluders who prefer $x$ to $c$. If $W_{x c} \neq \emptyset$, it builds a "profile" $\mathcal{Q}_{M \backslash W_{x c}}$ for $M \backslash W_{x c}$ s.t. the score of $x$ is $s\left(\mathcal{Q}_{M \backslash W_{x c}}, x\right)=0$, and the score of any other candidate $y \neq x$ is $s\left(\mathcal{Q}_{M \backslash W_{x c}}, y\right)=s_{1} \cdot\left|M \backslash W_{x c}\right|$. Finally, it solves the $R$-Coalitional Manipulation problem $\left(C, V \backslash W_{x c}, \mathcal{P}_{H} \cup \mathcal{Q}_{M \backslash W_{x c}}\right), x,\left|W_{x c}\right|$ to see whether the votes in $W_{x c}$ can be cast in order to make $x$ win the election. Next we prove that we manage to make $x$ win if and only if $x$ is $\alpha$-feasible for $W_{x c}$.

Claim. Let $\mathcal{Q}_{W_{x c}}$ be a profile. $x$ is the winner under $\mathcal{P}_{H} \cup \mathcal{Q}_{M \backslash W_{x c}} \cup \mathcal{Q}_{W_{x c}}$ (where $\mathcal{Q}_{M \backslash W_{x c}}$ is as defined above) if and only if for any (real) profile $\mathcal{P}_{M \backslash W_{x c}}^{\prime} x$ wins under $\mathcal{P}_{H} \cup \mathcal{P}_{M \backslash W_{x c}}^{\prime} \cup \mathcal{Q}_{W_{x c}}$.

\footnotetext{
${ }^{6}$ We used the double quotes for the word "profile" since it is not a real profile, but rather a score specification. Nevertheless, we use the above notation for simplicity, as if it were a real profile.
} 
Proof. We first prove the "only if" part. Suppose that $x$ wins under $\mathcal{P}_{H} \cup \mathcal{Q}_{M \backslash W_{x c}} \cup$ $\mathcal{Q}_{W_{x c}}$. Let $\mathcal{P}_{M \backslash W_{x c}}^{\prime}$ be any profile. For all $y, y \neq x$, it holds that $s\left(\mathcal{P}_{M \backslash W_{x c}}^{\prime}, y\right) \leq$ $s_{1} \cdot\left|M \backslash W_{x c}\right|$. Also, $s\left(\mathcal{P}_{M \backslash W_{x c}}^{\prime}, x\right) \geq 0$. Therefore, for each $y, y \neq x, s\left(\mathcal{P}_{H} \cup\right.$ $\left.\mathcal{P}_{M \backslash W_{x c}}^{\prime} \cup \mathcal{Q}_{W_{x c}}, x\right) \geq s\left(\mathcal{P}_{H} \cup \mathcal{Q}_{M \backslash W_{x c}} \cup \mathcal{Q}_{W_{x c}}, x\right)>s\left(\mathcal{P}_{H} \cup \mathcal{Q}_{M \backslash W_{x c}} \cup \mathcal{Q}_{W_{x c}}, y\right)=$ $s\left(\mathcal{P}_{H} \cup \mathcal{Q}_{W_{x c}}, y\right)+s_{1} \cdot\left|M \backslash W_{x c}\right| \geq s\left(\mathcal{P}_{H} \cup \mathcal{P}_{M \backslash W_{x c}}^{\prime} \cup \mathcal{Q}_{W_{x c}}, y\right) .^{7}$ And so, $x$ will win under $\mathcal{P}_{H} \cup \mathcal{P}_{M \backslash W_{x c}}^{\prime} \cup \mathcal{Q}_{W_{x c}}$.

Now we prove the "if" part. Suppose that for any profile $\mathcal{P}_{M \backslash W_{x c}}^{\prime}, x$ is the winner under $\mathcal{P}_{H} \cup \mathcal{P}_{M \backslash W_{x c}}^{\prime} \cup \mathcal{Q}_{W_{x c}}$. Let $y, y \neq x$ be a candidate. By our assumption, $x$ is the winner under $\mathcal{P}_{H} \cup \mathcal{P}_{M \backslash W_{x c}}^{\prime} \cup \mathcal{Q}_{W_{x c}}$, where $\mathcal{P}_{M \backslash W_{x c}}^{\prime}$ is built as follows: $y$ is ranked on top of each preference in $\mathcal{P}_{M \backslash W_{x c}}^{\prime}, x$ is ranked at the bottom of each preference in $\mathcal{P}_{M \backslash W_{x c}}^{\prime}$, and the other candidates are ranked arbitrarily. From this construction we get: $s\left(\mathcal{P}_{H} \cup \mathcal{Q}_{M \backslash W_{x c}} \cup \mathcal{Q}_{W_{x c}}, x\right)=s\left(\mathcal{P}_{H}, x\right)+s\left(\mathcal{Q}_{W_{x c}}, x\right)=s\left(\mathcal{P}_{H} \cup \mathcal{P}_{M \backslash W_{x c}}^{\prime} \cup\right.$ $\left.\mathcal{Q}_{W_{x c}}, x\right)>s\left(\mathcal{P}_{H} \cup \mathcal{P}_{M \backslash W_{x c}}^{\prime} \cup \mathcal{Q}_{W_{x c}}, y\right)=s\left(\mathcal{P}_{H}, y\right)+s_{1} \cdot\left|M \backslash W_{x c}\right|+s\left(\mathcal{Q}_{W_{x c}}, y\right)=$ $s\left(\mathcal{P}_{H} \cup \mathcal{Q}_{M \backslash W_{x c}} \cup \mathcal{Q}_{W_{x c}}, y\right)$. This is true for every $y \neq x$. Therefore, $x$ is the winner under $\mathcal{P}_{H} \cup \mathcal{Q}_{M \backslash W_{x c}} \cup \mathcal{Q}_{W_{x c}}$. (End of proof of claim.)

If we find a candidate $x \neq c$ and a non-empty coalition $W_{x c}$ such that $x$ is $\alpha$-feasible for $W_{x c}$, then $c$ is not in the $\alpha$-core. Otherwise, $c$ is in the $\alpha$-core.

Theorem 8. Let $k \in \mathbb{N}, 1 \leq k \leq|C|-1=m-1$. There is a polynomial-time algorithm solving the $k$-approval- $\beta$-Core problem.

Due to space limitations, the proof of Theorem 8 is omitted. ${ }^{8}$

Theorem 9. There exists a polynomial-time algorithm solving the Simplified-Bucklin$\alpha$-Core problem.

Proof. Suppose we are given an instance $I=(E, M, c)$ of the Bucklin- $\alpha$-Core problem, where $E=(C, V, \mathcal{P})$. The algorithm first checks whether $c \in F(M)$ by solving the Bucklin-Coalitional Manipulation problem (see [15] for the algorithm for this). It then goes over all the other candidates; for each candidate $x \neq c$ it computes the maximal set $W_{x c} \subseteq M$ of colluders who prefer $x$ to $c$. If $W_{x c} \neq \emptyset$, we need to check whether $x$ is $\alpha$-feasible for $W_{x c}$. We first introduce some new notation. Let $B(a, k, \mathcal{Q})$ denote the number of votes in the profile $\mathcal{Q}$ that rank the candidate $a$ in the $k$ first places. Let $d$ be the minimal integer such that $B\left(x, d, \mathcal{P}_{H}\right)+\left|W_{x c}\right|>\frac{1}{2} \cdot|V|$. Let $\mathcal{P}_{M \backslash W_{x c}}^{\prime \prime}$ be a "profile" of $M \backslash W_{x c}$ such that $B\left(x, d, \mathcal{P}_{M \backslash W_{x c}}^{\prime \prime}\right)=0$; and for all $y \neq x$, $B\left(y, 1, \mathcal{P}_{M \backslash W_{x c}}^{\prime \prime}\right)=\left|M \backslash W_{x c}\right| .^{9}$ In the following claim we prove that it is enough to check whether $x$ can win vs. $\mathcal{P}_{M \backslash W_{x c}}^{\prime \prime}$ to determine whether $x$ is $\alpha$-feasible for $W_{x c}$.

\footnotetext{
${ }^{7}$ We use here a strong inequality as we prove our results for the unique winner model. However, our results can be modified to work for the co-winner model as well.

${ }^{8}$ Again, see ftp://ftp.cs.huji.ac.il/users/jeff/winellzuckermanfull.pdf for a version of this paper containing the proof.

${ }^{9}$ Of course, if $m>2$ then $\mathcal{P}_{M \backslash W_{x c}}^{\prime \prime}$ cannot be a real profile; rather it is just a score specification. As before, we use the notation as if it were a real profile, for simplicity.
} 
Claim. $x$ is the winner under $\mathcal{P}_{H} \cup \mathcal{P}_{M \backslash W_{x c}}^{\prime \prime} \cup \mathcal{P}_{W_{x c}}^{x}$ where $\mathcal{P}_{M \backslash W_{x c}}^{\prime \prime}$ is as defined above, and $\mathcal{P}_{W_{x c}}^{x}$ is some fixed (real) profile for $W_{x c}$ which ranks $x$ in the first position in all the votes, if and only if for all profiles $\mathcal{P}_{M \backslash W_{x c}}^{\prime} x$ is the winner under $\mathcal{P}_{H} \cup \mathcal{P}_{M \backslash W_{x c}}^{\prime} \cup \mathcal{P}_{W_{x c}}^{x}$.

Proof. Suppose $x$ is the winner under $\mathcal{P}_{H} \cup \mathcal{P}_{M \backslash W_{x c}}^{\prime \prime} \cup \mathcal{P}_{W_{x c}}^{x}$. Let $\mathcal{P}_{M \backslash W_{x c}}^{\prime}$ be any profile. Let $d$ be as defined above. Since for all $y \neq x, B\left(y, d, \mathcal{P}_{M \backslash W_{x c}}^{\prime}\right) \leq\left|M \backslash W_{x c}\right|$, we have that $g_{y}^{\prime}:=\left\lfloor\frac{1}{2}|V|\right\rfloor-B\left(y, d, \mathcal{P}_{H} \cup \mathcal{P}_{M \backslash W_{x c}}^{\prime}\right) \geq\left\lfloor\frac{1}{2}|V|\right\rfloor-B\left(y, d, \mathcal{P}_{H}\right)-\mid M \backslash$ $W_{x c} \mid=\left\lfloor\frac{1}{2}|V|\right\rfloor-B\left(y, d, \mathcal{P}_{H} \cup \mathcal{P}_{M \backslash W_{x c}}^{\prime \prime}\right)=: g_{y}^{\prime \prime}$. Therefore, $k_{y}^{\prime}:=\min \left\{g_{y}^{\prime},\left|W_{x c}\right|\right\} \geq$ $\min \left\{g_{y}^{\prime \prime},\left|W_{x c}\right|\right\}=: k_{y}^{\prime \prime}$. Since $x$ is the winner under $\mathcal{P}_{H} \cup \mathcal{P}_{M \backslash W_{x c}}^{\prime \prime} \cup \mathcal{P}_{W_{x c}}^{x}$, we have for all $y \neq x, B\left(y, d, \mathcal{P}_{W_{x c}}^{x}\right) \leq k_{y}^{\prime \prime} \leq k_{y}^{\prime}$. So, by definition of $k_{y}^{\prime}, B\left(y, d, \mathcal{P}_{H} \cup \mathcal{P}_{M \backslash W_{x c}}^{\prime} \cup\right.$ $\left.\mathcal{P}_{W_{x c}}^{x}\right) \leq\left\lfloor\frac{1}{2}|V|\right\rfloor$. On the other hand, $B\left(x, d, \mathcal{P}_{H} \cup \mathcal{P}_{M \backslash W_{x c}}^{\prime} \cup \mathcal{P}_{W_{x c}}^{x}\right) \geq B\left(x, d, \mathcal{P}_{H} \cup\right.$ $\left.\mathcal{P}_{W_{x c}}^{x}\right)=B\left(x, d, \mathcal{P}_{H}\right)+\left|W_{x c}\right|>\frac{1}{2}|V|$ (where the last inequality follows from the definition of $d$ ). And so, $x$ wins under $\mathcal{P}_{H} \cup \mathcal{P}_{M \backslash W_{x c}}^{\prime} \cup \mathcal{P}_{W_{x c}}^{x}$.

For the opposite direction, suppose that for every profile $\mathcal{P}_{M \backslash W_{x c}}^{\prime}, x$ is the winner under $\mathcal{P}_{H} \cup \mathcal{P}_{M \backslash W_{x c}}^{\prime} \cup \mathcal{P}_{W_{x c}}^{x}$, where $\mathcal{P}_{W_{x c}}^{x}$ is some fixed profile of $W_{x c}$ with $x$ ranked at the top of each vote. Let $y, y \neq x$, be any candidate. $\mathcal{P}_{W_{x c}}^{x}$ makes $x$ win also vs. the profile $\mathcal{P}_{M \backslash W_{x c}}^{y, x}$ where $y$ is ranked first and $x$ is ranked last in all the preferences. Since the score of $x$ under $\mathcal{P}_{H} \cup \mathcal{P}_{M \backslash W_{x c}}^{y, x} \cup \mathcal{P}_{W_{x c}}^{x}$ is $d$, it follows that the score of $y$ under $\mathcal{P}_{H} \cup$ $\mathcal{P}_{M \backslash W_{x c}}^{y, x} \cup \mathcal{P}_{W_{x c}}^{x}$ is greater than $d$. Therefore, $B\left(y, d, \mathcal{P}_{W_{x c}}^{x}\right) \leq \min \left\{\left|W_{x c}\right|,\left\lfloor\frac{1}{2}|V|\right\rfloor-\right.$ $\left.B\left(y, d, \mathcal{P}_{H}\right)-\left|M \backslash W_{x c}\right|\right\}$. Hence, the score of $y$ under $\mathcal{P}_{H} \cup \mathcal{P}_{M \backslash W_{x c}}^{\prime \prime} \cup \mathcal{P}_{W_{x c}}^{x}$ is $>d$. On the other hand, by definition of $d$ and $\mathcal{P}_{W_{x c}}^{x}, B\left(x, d, \mathcal{P}_{H} \cup \mathcal{P}_{M \backslash W_{x c}}^{\prime \prime} \cup \mathcal{P}_{W_{x c}}^{x}\right)=$ $B\left(x, d, \mathcal{P}_{H} \cup \mathcal{P}_{W_{x c}}^{x}\right)=B\left(x, d, \mathcal{P}_{H}\right)+\left|W_{x c}\right|>\frac{1}{2} \cdot|V|$. So, the score of $x$ under $\mathcal{P}_{H} \cup \mathcal{P}_{M \backslash W_{x c}}^{\prime \prime} \cup \mathcal{P}_{W_{x c}}^{x}$ is $d$. Hence, $x$ is the winner under $\mathcal{P}_{H} \cup \mathcal{P}_{M \backslash W_{x c}}^{\prime \prime} \cup \mathcal{P}_{W_{x c}}^{x}$. (End of proof of claim.)

We now resume the proof of Theorem 9. With the above claim in hand, we can compute in polynomial time whether $x$ is $\alpha$-feasible for the coalition $W_{x c}$, in the following way. First compute $d$ as the minimal integer such that $B\left(x, d, \mathcal{P}_{H}\right)+\left|W_{x c}\right|>$ $\frac{1}{2}|V|$. Then define for each $y \neq x, g_{y}^{\prime \prime}=\left\lfloor\frac{1}{2}|V|\right\rfloor-B\left(y, d, \mathcal{P}_{H}\right)-\left|M \backslash W_{x c}\right|$, and $k_{y}^{\prime \prime}=\min \left\{g_{y}^{\prime \prime},\left|W_{x c}\right|\right\}$. If $\sum_{y \neq x} k_{y}^{\prime \prime}<(d-1)\left|W_{x c}\right|$, then there does not exist a profile $\mathcal{P}_{W_{x c}}^{\prime \prime \prime}$ making $x$ win under $\mathcal{P}_{H} \cup \mathcal{P}_{M \backslash W_{x c}}^{\prime \prime} \cup \mathcal{P}_{W_{x c}}^{\prime \prime \prime}$ (see [15] for details of the algorithm). Otherwise, we build the profile $\mathcal{P}_{W_{x c}}^{x}$ as follows. We first put $x$ on top of all the preferences of $\mathcal{P}_{W_{x c}}^{x}$. Then for all $i=1, \ldots, m-1$ we put the candidate $c_{i}$ in the next $k_{c_{i}}^{\prime \prime}$ available places in the votes of $\mathcal{P}_{W_{x c}}^{x}$, such that $B\left(c_{i}, d, \mathcal{P}_{W_{x c}}^{x}\right) \leq k_{c_{i}}^{\prime \prime}$, until we fill all the critical places. By Claim 4, if we have found a manipulation, then it works for all profiles $\mathcal{P}_{M \backslash W_{x c}}^{\prime}$, and if we have not found a manipulation, then there does not exist a manipulation that works for all the profiles $\mathcal{P}_{M \backslash W_{x c}}^{\prime}$. That is, we have found a manipulation if and only if $x \in F_{\alpha}\left(W_{x c}\right)$. If we have found some $x \neq c$ such that $W_{x c} \neq \emptyset$ and $x \in F_{\alpha}\left(W_{x c}\right)$, then $c$ is not in the $\alpha$-core. Otherwise, $c$ is in the $\alpha$-core.

Theorem 10. There exists a polynomial-time algorithm solving the Simplified-Bucklin$\beta$-Core problem. 
The proof of Theorem 10 is omitted due to space limitations. ${ }^{10}$

Theorem 11. Let $R$ be the Plurality with Runoff voting rule. There exists a polynomialtime algorithm solving the $R$ - $\beta$-Core problem.

Proof. Suppose we are given an instance $I=(E, M, c)$ of the $R$ - $\beta$-Core problem, where $R$ is the Plurality with Runoff voting rule, and $E=(C, V, \mathcal{P})$. The algorithm first checks whether $c \in F(M)$ by solving the $R$-Coalitional Manipulation problem (see [17] for the algorithm for this). Then it goes over all the other candidates. For each candidate $x \neq c$ it computes the maximal set $W_{x c} \subseteq M$ of the colluders who prefer $x$ to $c$. If $W_{x c} \neq \emptyset$, we need to check whether $x$ is $\beta$-feasible for $W_{x c}$. To do so, we iterate over all candidates $a \neq x$, and for each $a$, we check whether $W_{x c}$ can make $x$ win vs. the profile $\mathcal{P}_{M \backslash W_{x c}}^{a, x}$ where everybody ranks $a$ first, $x$ last, and the other candidates in some arbitrary order. Then we iterate over all pairs of candidates $(a, b)$, and for each pair $(a, b)$ and for all $i=1, \ldots,\left|M \backslash W_{x c}\right|-1$, we check whether $W_{x c}$ can make $x$ win vs. the profile $\mathcal{P}_{M \backslash W_{x c}}^{i, a, b, x}$ where $i$ voters in $M \backslash W_{x c}$ rank $a$ first, the $\left|M \backslash W_{x c}\right|-i$ remaining voters rank $b$ first, all these voters rank $x$ last, and the other candidates are ranked in some arbitrary order. In the next claim we prove that it is enough to check these profiles to determine whether $x$ is $\beta$-feasible for $W_{x c}$.

Claim. If:

1. for each $a \neq x$, there exists a profile $\mathcal{P}_{W_{x c}}^{\prime \prime}$ such that $x$ is the winner under $\mathcal{P}_{H} \cup$ $\mathcal{P}_{M \backslash W_{x c}}^{a, x} \cup \mathcal{P}_{W_{x c}}^{\prime \prime}$, and

2. for each pair $(a, b)$ (where $x \notin\{a, b\}$ ) and for each $i, 1 \leq i \leq\left|M \backslash W_{x c}\right|-1$, there exists a profile $\mathcal{P}_{W_{x c}}^{\prime \prime \prime}$ such that $x$ is the winner under $\mathcal{P}_{H} \cup \mathcal{P}_{M \backslash W_{x c}}^{i, a, b, x} \cup \mathcal{P}_{W_{x c}}^{\prime \prime \prime}$,

then $x$ is $\beta$-feasible. Otherwise $x$ is not $\beta$-feasible.

Proof. It is clear that if (1) there is a candidate $a \neq x$, such that for each profile $\mathcal{P}_{W_{x c}}^{\prime}$ $x$ is not a winner under $\mathcal{P}_{H} \cup \mathcal{P}_{M \backslash W_{x c}}^{a, x} \cup \mathcal{P}_{W_{x c}}^{\prime}$, or (2) there is a pair of candidates $(a, b)$ and $i, 1 \leq i \leq\left|M \backslash W_{x c}\right|-1$, such that for each profile $\mathcal{P}_{W_{x c}}^{\prime} x$ is not a winner under $\mathcal{P}_{H} \cup \mathcal{P}_{M \backslash W_{x c}}^{i, a, b, x} \cup \mathcal{P}_{W_{x c}}^{\prime}$, then by definition, $x$ is not $\beta$-feasible for $W_{x c}$. For the other direction, suppose that for each $a \neq x$, there exists a profile $\mathcal{P}_{W_{x c}}^{\prime \prime}$ such that $x$ is the winner under $\mathcal{P}_{H} \cup \mathcal{P}_{M \backslash W_{x c}}^{a, x} \cup \mathcal{P}_{W_{x c}}^{\prime \prime}$, and for each $(a, b)$ and $i$ there exists a profile $\mathcal{P}_{W_{x c}}^{\prime \prime \prime}$ such that $x$ is the winner under $\mathcal{P}_{H} \cup \mathcal{P}_{M \backslash W_{x c}}^{i, a, b, x} \cup \mathcal{P}_{W_{x c}}^{\prime \prime \prime}$. Let $\mathcal{P}_{M \backslash W_{x c}}^{\prime}$ be any profile for $M \backslash W_{x c}$. We will show that there exists a profile $\mathcal{P}_{W_{x c}}^{\prime}$ such that $x$ is the winner of the election under $\mathcal{P}_{H} \cup \mathcal{P}_{M \backslash W_{x c}}^{\prime} \cup \mathcal{P}_{W_{x c}}^{\prime}$. Let $y, z \in C$ be the winners of the first round under the partial profile $\mathcal{P}_{H} \cup \mathcal{P}_{M \backslash W_{x c}}^{\prime}$. Denote by $\gamma(\mathcal{P}, b)$ the plurality score of candidate $b$ under the profile $\mathcal{P}$. Suppose w.l.o.g. that

$$
\gamma\left(\mathcal{P}_{H} \cup \mathcal{P}_{M \backslash W_{x c}}^{\prime}, y\right) \geq \gamma\left(\mathcal{P}_{H} \cup \mathcal{P}_{M \backslash W_{x c}}^{\prime}, z\right) .
$$

Denote by $N(\mathcal{P}, a, b)$ the number of votes in the profile $\mathcal{P}$ who prefer $a$ over $b$. We divide the proof into 3 cases:

\footnotetext{
${ }^{10}$ See ftp://ftp.cs.huji.ac.il/users/jeff/winellzuckermanfull.pdf for a version of this paper containing the proof.
} 
Case 1. $x \notin\{y, z\}$. Let us consider the profile $\mathcal{P}_{M \backslash W_{x c}}^{i, y, z, x}$, where $i=\left|M \backslash W_{x c}\right|-$ $\gamma\left(\mathcal{P}_{M \backslash W_{x c}}^{\prime}, z\right)$. That is, we choose $i$ such that $\gamma\left(\mathcal{P}_{M \backslash W_{x c}}^{i, y, z, x}, z\right)=\gamma\left(\mathcal{P}_{M \backslash W_{x c}}^{\prime}, z\right)$. Let $\mathcal{P}_{W_{x c}}^{\prime \prime \prime}$ be the profile such that $x$ is the winner under $\mathcal{P}_{H} \cup \mathcal{P}_{M \backslash W_{x c}}^{i, y, z, x} \cup \mathcal{P}_{W_{x c}}^{\prime \prime \prime}$. Let $x$ and $b$ be the two candidates who proceed to the second round under $\mathcal{P}_{H} \cup \mathcal{P}_{M \backslash W_{x c}}^{i, y, z, x} \cup \mathcal{P}_{W_{x c}}^{\prime \prime \prime}$. Here we have 3 cases:

Case 1.a. $b=y$. We may assume that $\mathcal{P}_{W_{x c}}^{\prime \prime \prime}$ has in the first places only $x$ 's and $y$ 's (if it does not, then we can change it appropriately, and the winners in the first and the second round will not change). Then under $\mathcal{P}_{H} \cup \mathcal{P}_{M \backslash W_{x c}}^{\prime} \cup \mathcal{P}_{W_{x c}}^{\prime \prime \prime}$ the only possible winners of the first round are $y, z$ and $x$. Denote for brevity, $\mathcal{Q}^{1}=\mathcal{P}_{H} \cup$ $\mathcal{P}_{M \backslash W_{x c}}^{\prime} \cup \mathcal{P}_{W_{x c}}^{\prime \prime \prime}$, and $\mathcal{Q}^{2}=\mathcal{P}_{H} \cup \mathcal{P}_{M \backslash W_{x c}}^{i, y, z, x} \cup \mathcal{P}_{W_{x c}}^{\prime \prime \prime}$. Since $x$ is ranked last by all the voters in $\mathcal{P}_{M \backslash W_{x c}}^{i, y, z, x}$, we have $\gamma\left(\mathcal{Q}^{1}, x\right) \geq \gamma\left(\mathcal{Q}^{2}, x\right)$. Also, by the definition of $\mathcal{P}_{M \backslash W_{x c}}^{i, y, z, x}$, we have $\gamma\left(\mathcal{Q}^{1}, y\right) \leq \gamma\left(\mathcal{Q}^{2}, y\right)$ and $\gamma\left(\mathcal{Q}^{1}, z\right)=\gamma\left(\mathcal{Q}^{2}, z\right)$. As mentioned before, $x$ is one of the winners of the first round under $\mathcal{Q}^{2}$. It follows that also under the profile $\mathcal{Q}^{1} x$ will be one of the winners of the first round. By assumption (1), and by the inequality $\gamma\left(\mathcal{P}_{W_{x c}}^{\prime \prime \prime}, y\right) \geq \gamma\left(\mathcal{P}_{W_{x c}}^{\prime \prime \prime}, z\right)$, we have $\gamma\left(\mathcal{Q}^{1}, y\right) \geq \gamma\left(\mathcal{Q}^{1}, z\right)$, and so the second winner of the first round is $y$. Now, $N\left(\mathcal{P}_{M \backslash W_{x c}}^{\prime}, x, y\right) \geq 0=N\left(\mathcal{P}_{M \backslash W_{x c}}^{i, y, z, x}, x, y\right)$. So, since $x$ beats $y$ in the second round under the profile $\mathcal{Q}^{2}, x$ also beats $y$ in the second round under $\mathcal{Q}^{1}$. So, we found a profile $\left(\mathcal{P}_{W_{x c}}^{\prime \prime \prime}\right)$, such that $x$ is the winner under $\mathcal{Q}^{1}=$ $\mathcal{P}_{H} \cup \mathcal{P}_{M \backslash W_{x c}}^{\prime} \cup \mathcal{P}_{W_{x c}}^{\prime \prime \prime}$.

Case 1.b. $b=z$. Here we may assume that $\mathcal{P}_{W_{x c}}^{\prime \prime \prime}$ contains in the first places only $x$ 's and $z$ 's. Here we also have that the only possible winners of the first round under $\mathcal{Q}^{1}$ are $x, y$ and $z$. Again, we have $\gamma\left(\mathcal{Q}^{1}, x\right) \geq \gamma\left(\mathcal{Q}^{2}, x\right), \gamma\left(\mathcal{Q}^{1}, y\right) \leq \gamma\left(\mathcal{Q}^{2}, y\right)$ and $\gamma\left(\mathcal{Q}^{1}, z\right)=\gamma\left(\mathcal{Q}^{2}, z\right)$. So, since under $\mathcal{Q}^{2}$ the winners of the first round are $x$ and $z$, we have that under $\mathcal{Q}^{1}$ the winners are also $x$ and $z . x$ beats $z$ in the second round under $\mathcal{Q}^{2}$, and $N\left(\mathcal{P}_{M \backslash W_{x c}}^{\prime}, x, z\right) \geq 0=N\left(\mathcal{P}_{M \backslash W_{x c}}^{i, y, z, x}, x, z\right)$. Hence, $x$ beats $z$ in the second round under $\mathcal{Q}^{1}$ as well.

Case 1.c. $b \notin\{y, z\}$. We may assume that $\mathcal{P}_{W_{x c}^{\prime \prime \prime}}$ contains in the first places only $x$ 's and $b$ 's. The only possible winners of the first round under $\mathcal{Q}^{1}$ are $y, z, x$ and $b$. We have the following inequalities: $\gamma\left(\mathcal{P}_{M \backslash W_{x c}}^{\prime}, x\right) \geq 0=\gamma\left(\mathcal{P}_{M \backslash W_{x c}}^{i, y, z, x}, x\right), \gamma\left(\mathcal{P}_{M \backslash W_{x c}}^{\prime}, b\right) \geq 0=$ $\gamma\left(\mathcal{P}_{M \backslash W_{x c}}^{i, y, z, x}, b\right), \gamma\left(\mathcal{P}_{M \backslash W_{x c}}^{\prime}, y\right) \leq \gamma\left(\mathcal{P}_{M \backslash W_{x c}}^{i, y, z, x}, y\right)$, and $\gamma\left(\mathcal{P}_{M \backslash W_{x c}}^{\prime}, z\right)=\gamma\left(\mathcal{P}_{M \backslash W_{x c}}^{i, y, z, x}, z\right)$. And so, since $x$ and $b$ are the winners of the first round under $\mathcal{Q}^{2}$, they are also the winners of the first round under $\mathcal{Q}^{1}$. In the second round, $x$ beats $b$ under the profile $\mathcal{Q}^{2}$, and $N\left(\mathcal{P}_{M \backslash W_{x c}}^{\prime}, x, b\right) \geq 0=N\left(\mathcal{P}_{M \backslash W_{x c}}^{i, y, z, x}, x, b\right)$. It follows that $x$ beats $b$ in the second round under the profile $\mathcal{Q}^{1}$ as well.

Case 2. $x=y$. So $x$ and $z$ are the two winners of the first round under the partial profile $\mathcal{P}_{H} \cup \mathcal{P}_{M \backslash W_{x c}}^{\prime}$. Recall that $\mathcal{P}_{M \backslash W_{x c}}^{z, x}$ is a profile where everybody in $M \backslash W_{x c}$ ranks $z$ first, $x$ last, and the other candidates in some arbitrary order. Let $\mathcal{P}_{W_{x c}}^{\prime \prime}$ be the profile such that $x$ is the winner under $\mathcal{P}_{H} \cup \mathcal{P}_{M \backslash W_{x c}}^{z, x} \cup \mathcal{P}_{W_{x c}}^{\prime \prime}$. Denote, for shortness, $\mathcal{Q}^{3}=\mathcal{P}_{H} \cup \mathcal{P}_{M \backslash W_{x c}}^{\prime} \cup \mathcal{P}_{W_{x c}}^{\prime \prime}$ and $\mathcal{Q}^{4}=\mathcal{P}_{H} \cup \mathcal{P}_{M \backslash W_{x c}}^{z, x} \cup \mathcal{P}_{W_{x c}}^{\prime \prime}$. Let $x$ and $d$ be the two candidates who are the winners of the first round under $\mathcal{Q}^{4}$.

Case 2.a. $d=z$. Here we can assume that $\mathcal{P}_{W_{x c}}^{\prime \prime}$ contains only $x$ 's and $z$ 's in the first places. Hence, under $\mathcal{Q}^{3}, x$ and $z=d$ are the winners of the first round. 
Case 2.b. $d \neq z$. We can assume that $\mathcal{P}_{W_{x c}}^{\prime \prime}$ contains only $x$ 's and $d$ 's in the first places. Therefore, under $\mathcal{Q}^{3}$ the only possible winners of the first round are $x, z$ and $d$. Since under $\mathcal{Q}^{4} x$ and $d$ proceed to the second round, and due to the fact that in $\mathcal{P}_{M \backslash W}^{z, x}$ $z$ is ranked on top of all the preferences, we have $\gamma\left(\mathcal{Q}^{3}, d\right) \geq \gamma\left(\mathcal{Q}^{4}, d\right)>\gamma\left(\mathcal{Q}^{4}, z\right) \geq$ $\gamma\left(\mathcal{Q}^{3}, z\right)$, and $\gamma\left(\mathcal{Q}^{3}, x\right) \geq \gamma\left(\mathcal{Q}^{4}, x\right)>\gamma\left(\mathcal{Q}^{4}, z\right) \geq \gamma\left(\mathcal{Q}^{3}, z\right)$. Therefore, under $\mathcal{Q}^{3}, x$ and $d$ are the winners of the first round.

Case 2 (continued). Now, as $x$ is the winner of the second round under $\mathcal{Q}^{4}$, and since $N\left(\mathcal{P}_{M \backslash W_{x c}}^{\prime}, x, d\right) \geq 0=N\left(\mathcal{P}_{M \backslash W_{x c}}^{z, x}, x, d\right)$, we have $N\left(\mathcal{Q}^{3}, x, d\right) \geq N\left(\mathcal{Q}^{4}, x, d\right)>$ $N\left(\mathcal{Q}^{4}, d, x\right) \geq N\left(\mathcal{Q}^{3}, d, x\right)$. Hence, $x$ beats $d$ in the second round under $\mathcal{Q}^{3}$, and so $x$ wins the election.

Case 3. $x=z$. This case is handled similarly to Case 2. (End of proof of claim.)

If we found $x \neq c$ such that $W_{x c} \neq \emptyset$ and $x \in F_{\beta}\left(W_{x c}\right)$, then by definition $c \notin \beta$ core. Otherwise, $c \in \beta$-core.

\section{Conclusions and Future Work}

In this paper we have provided a computational analysis of the following question: given a coalition of manipulative voters, which candidate should they manipulate in favor of, given that they might not have identical preferences. To perform our analysis we have used the notions of $\alpha$ - and $\beta$-core, which-under various assumptions-describe the sets of candidates that the manipulators can support in a stable manner (i.e., without running the risk of breaking the coalition).

Our main results are the following. The complexity of determining membership in the $\alpha$-and $\beta$-cores is at least as high as the complexity of the constructive coalitional manipulation problem for the same rule. On the other hand, for the several prominent voting rules for which coalitional manipulation is easy, we have also provided polynomial time algorithms for determining membership in the $\alpha$ - and $\beta$-cores. One direction for future work is to extend the above research to other voting rules. Another interesting direction is to try to find voting rules that produce an outcome in the core, if the core is non-empty, when applied to the truthful preferences of the voters. Yet another direction is to investigate the computational complexity of finding a voting profile of the colluders which is a strong Nash equilibrium, if one exists.

\section{Acknowledgments}

Michael Zuckerman and Jeffrey S. Rosenschein were partially funded by Israel Science Foundation grant \#898/05, Israel Ministry of Science and Technology grant \#3-6797, and, along with Vincent Conitzer, United States-Israel Binational Science Foundation grant \#2006-216. Piotr Faliszewski was Supported by AGH University of Technology Grant no. 11.11.120.865, by Polish Ministry of Science and Higher Education grant N-N206-378637, and by Foundation for Polish Science's program Homing/Powroty. Vincent Conitzer acknowledges NSF IIS-0812113, IIS-0953756, and CCF-1101659, as well as an Alfred P. Sloan fellowship, for support. 


\section{References}

1. R. Aumann. Acceptable points in general cooperative n-person games. Contributions to the Theory of Games, 4:287-324, 1959.

2. Y. Bachrach, E. Elkind, and P. Faliszewski. Coalitional voting manipulation: A gametheoretic perspective. In The Twenty-Second International Joint Conference on Artificial Intelligence (IJCAI 2011), pages 49-54, 2011.

3. J. Bartholdi and J. Orlin. Single Transferable Vote resists strategic voting. Social Choice and Welfare, 8:341-354, 1991.

4. J. Bartholdi, C. A. Tovey, and M. A. Trick. The computational difficulty of manipulating an election. Social Choice and Welfare, 6:227-241, 1989.

5. V. Conitzer, T. Sandholm, and J. Lang. When are elections with few candidates hard to manipulate? JACM, 54(3), June 2007.

6. P. Faliszewski, E. Hemaspaandra, and L. Hemaspaandra. How hard is bribery in elections? Journal of AI Research, 35:485-532, 2009.

7. P. Faliszewski, E. Hemaspaandra, and L. Hemaspaandra. Using complexity to protect elections. Communications of the ACM, 53(11):74-82, 2010.

8. P. Faliszewski and A. Procaccia. AI's war on manipulation: Are we winning? AI Magazine, 31(4):53-64, 2010.

9. A. Gibbard. Manipulation of voting schemes. Econometrica, 41:587-602, 1973.

10. S. Obraztsova and E. Elkind. On the complexity of voting manipulation under randomized tie-breaking. In The Twenty-Second International Joint Conference on Artificial Intelligence (IJCAI 2011), pages 319-324, 2011.

11. S. Obraztsova, E. Elkind, and N. Hazon. Ties matter: Complexity of voting manipulation revisited. In The Tenth International Joint Conference on Autonomous Agents and Multiagent Systems (AAMAS 2011), pages 71-78, 2011.

12. B. Peleg and P. Sudholter. Introduction to the Theory of Cooperative Games. Kluwer Academic Publishers, Boston, 2003.

13. M. Satterthwaite. Strategy-proofness and Arrow's conditions: Existence and correspondence theorems for voting procedures and social welfare functions. Journal of Economic Theory, 10:187-217, 1975.

14. L. Xia, V. Conitzer, and A. D. Procaccia. A scheduling approach to coalitional manipulation. In Proceedings of the 11th ACM Conference on Electronic Commerce (EC'10), pages 275284, 2010.

15. L. Xia, M. Zuckerman, A. D. Procaccia, V. Conitzer, and J. S. Rosenschein. Complexity of unweighted coalitional manipulation under some common voting rules. In The TwentyFirst International Joint Conference on Artificial Intelligence (IJCAI 2009), pages 348-353, Pasadena, California, July 2009.

16. M. Zuckerman, O. Lev, and J. S. Rosenschein. An algorithm for the coalitional manipulation problem under maximin. In The Tenth International Joint Conference on Autonomous Agents and Multiagent Systems (AAMAS 2011), pages 845-852, Taipei, Taiwan, 2011.

17. M. Zuckerman, A. D. Procaccia, and J. S. Rosenschein. Algorithms for the coalitional manipulation problem. AI Journal, 173(2):392-412, February 2009. 